into the present study from January 2006 to July 2009. Demographic information, concomitant diseases, peri-operative laboratory examinations, angiographic features, and surgery information of consecutive patients who underwent PCI were collected.

Results A total of 13922 patients were recorded in the database, of which $9.03 \%(1257 / 13,922)$ had previous PCI. Univariate analysis revealed that patients with prior PCI had significant higher prevalence of hypertension, diabetes mellitus and hyperlipidaemia than those without prior PCI. In addition, the percentage of patients with prior $C A B G$ was higher in the former group (6.0\% vs $1.9 \%$, $\mathrm{p}<0.001$ ). In multivariable logistic regression analyses adjusted for demographic, clinical, angiographic and procedural factors, elderly age (OR $1.01,95 \%$ CI 1.00 to 1.01), male (OR 1.74, 95\% CI 1.48 to 2.04), hypertension (OR $1.36,95 \%$ CI 1.19 to 1.54 ), diabetes mellitus (OR $1.43,95 \%$ CI 1.24 to 1.63 ), hyperlipidaemia (OR $1.30,95 \% \mathrm{CI}$ 1.06 to 1.57 ) and prior CABG (OR 3.37, 95\% CI 2.52 to 4.51) were identified as independent risk factors of secondary PCI. Additionally, history of prior $\mathrm{CABG}$ was the most important predictor of secondary PCI.

Conclusions Risk factors associated with secondary PCI include elderly age, male, hypertension, diabetes mellitus, hyperlipidaemia and prior $\mathrm{CABG}$, of which previous $\mathrm{CABG}$ was the most important.

\section{e0417 EFFECT OF SHENGMAI INJECTION ON CARDIAC FUNCTION AND INFLAMMATORY REACTION IN PATIENTS WITH ACUTE CORONARY SYNDROME}

doi:10.1136/hrt.2010.208967.417

Zhang Yachen, Chen Man-tian, Wang Fei, Rong Yezhi, Lu Baojing. Division of Cardiology, Xinhua Hospital, School of Medicine, Shanghai Jiaotong University

Objective To confirm the effect of Shengmai injection in improving cardiac function in patients with acute coronary syndrome (ACS) and to explore its influence on inflammatory reaction in patients. Methods Ninety ACS patients were randomised into two groups, the control group, treated with conventional therapy and the SMI group, treated with SMI. The patients' cardiac function was noted and the high sensitive C-reactive protein (hs-CRP) in venous blood was measured before treatment and 1 week and 2 weeks after treatment, so as to observe and compare their changes in the two groups.

Results The cardiac output, stroke volume and ejection fraction in the SMI group after 3 weeks of treatment were all higher than those in the control group $(p<0.05)$. The serum content of hsCRP was reduced in both groups, but the reduction in the SMI group was more significant than that in the control group.

Conclusion SMI could improve cardiac function and further inhibit the inflammatory reaction in patients with ACS.

\section{e0418 EFFECT OF EECP-INTEGRATED STANDARD THERAPY FOR PATIENTS WITH STABLE CORONARY ARTERY DISEASE: A PROSPECTIVE, RANDOMISED, OPEN-LABELLED AND BLIND-ENDPOINT STUDY (PROBE-EECP TRIAL)}

doi:10.1136/hrt.2010.208967.418

Du Zhimin, Wu Guifu, Xie Qiang, Zhang Yan, Yang Daya, Ling Li, Liu Donghong, Tang Lilong, Chen Guangye, Ma Hong. Division of Cardiology The First Affiliated Hospital of Sun Yat-sen University Guangzhou

Background Enhanced External Counterpulsation (EECP) has been introduced as an noninvasive, effective and safe therapy for the management of ischaemic vascular diseases. Previously, researches had been focused on the mechanisms of EECP on vascular diseases. However, clinical data in this area are still relatively in paucity.
Objective To determine whether EECP-integrated standard therapy would reduce the major adverse cardiovascular events in patients with coronary artery diseases and improve the quality of life.

Method A total of 194 patients aged from 32 to 75 years old with coronary artery diseases from Sept. 2008 to Dec. 2009 in 4 hospitals affiliated to Sun Yat-sen University were enrolled into the trial. Subjects were randomised to be allocated either to EECP plus standard therapy group or standard therapy group, and followed for 0.5 to 1.5 -year. The coronary artery disease in all patients was documented by coronary angiography, or a history of prior myocardial infarction or prior coronary revascularization. Patients in standard therapy group were treated with guideline-driven therapeutic strategy, and patients in EECP group were given $36 \mathrm{~h}$ of EECP in addition to the guideline-driven therapeutic strategy. Repeated EECP were prescribed to patients with any reoccurred ischaemic symptoms, or new ischaemia, or no symptoms in 12 months later. Follow-up visits were performed at 1, 3, 6, 12 months and each year therefore from the inclusion.

Results The primary composite endpoints of myocardial infarction, revascularisation, readmission to hospital due to stroke and ACS occurred in 6 of $104(5.77 \%)$ patients in EECP group compared with 9 of $91(9.89 \%)$ in standard therapy group $(p<0.05)$. There was 1 death in both groups respectively. The incidence of minor skin damage was about $8 \%$ in EECP group, causing EECP therapy in 3 patients to be prematurely terminated.

Conclusion An EECP-integrated standard therapy significantly reduced major cardiovascular events in patients with documented coronary artery disease.

\section{e0419 THE APPLYING OF ASPIRATION CATHETER IN REVASCULARISATION IN PATIENTS WITH ST-ELEVATION MYOCARDIAL INFARCTION}

doi:10.1136/hrt.2010.208967.419

Li Weiming, Xu Yawei, Wang Ke, Chen Yangqing, Wei Yidong, Li Yuanmin, Che Wenliang, Hou Lei, Lu Yunlan. 'Department of Cardiologyshanghai Tenth People's Hospital

Objectives To observe the immediate and recent treatment effects of applying the aspiration catheter in patients with ST-elevation myocardial infarction (STEMI) undergoing primary percutaneous coronary intervention (PCI).

Methods From March to June 2010, we enrolled the STEMI patients presenting with TIMI Flow Grade 0 or 1 in the infarct related artery (IRA) at baseline CAG undergoing primary PCI. The aspiration catheter (Medtronic Inc., Export?) was applied immediately to aspirate the intracoronary thrombus. Whether predilatating and/or stenting were decided by the blood flow and the condition of lesions. The patients from October 2009 to February 2010 who was diagnosed as STEMI and received non-aspiration were enrolled as control group. The immediate and recent clinical outcomes of applying the aspiration catheter were compared between the two groups.

Results There were 25 cases in Group thrombus-aspiration (2 cases of subacute stent thrombosis). After having aspirated the intracoronary thrombus using the aspiration catheter, 14 cases of STEMI recovered immediately $(9$ cases received direct stenting; 3 cases implanted stents after balloon predilatation, and had satisfied results; 2 cases of subacute stent thrombosis received the antithrombotic therapy, included intravenous infusion of Xinweining for $36 \mathrm{~h}$ in CCU immediately after recovering TIMI grade- 3 flow). 10 cases recovered TIMI 1-2, 1 cases also showed no-reflow. All of the 11 cases received the balloon predilatation and stenting, only one showed slow flow, the others recovered. There was no other severe complication during and after the operation. There was no in-stent thrombosis during 1 month follow-up, and the cardiac function improved largely. There 
were 22 cases in Group non-aspiration, 4 cases received direct stenting, 18 cases received balloon predilatation and stenting. 5 cases showed slow flow, 3 cases recovered normal flow after intra-coronary infusion of nitroglycerin, verapamil and Xinweinin, but 2 cases also showed TIMI 1, and accompanied heart failure.

Conclusions The applying of aspiration catheter in patients with STEMI prior to primary PCI could increase the opportunities of direct stenting, improve myocardial reperfusion, immediate and recent clinical outcomes as compared with PCI in the absence of thrombus aspiration, and also show ease and safe procedure.

\section{e0420 C-REACTIVE PROTEIN FOR PREDICTING CLINICAL OUTCOMES AFTER DRUG-ELUTING STENT IMPLANTATION}

doi:10.1136/hrt.2010.208967.420

Yu Miao, Zhou Yujie, Wang Zhijian, Shi Dongmei, Liu Yuyang, Zhao Yingxin, Guo Yonghe. Anzhen Hospital Capital Medical University

Background Although C-reactive protein (CRP) has been proposed as a useful biomarker for predicting atherothrombosis, the association of CRP levels and clinical outcomes after drug-eluting stent (DES) implantation has not been defined.

Objectives To assess the predictive value of CRP after drug-eluting stent (DES) implantation, especially the association of CRP levels with adverse clinical events, such as stent thrombosis, death, myocardial infarction (MI) and so on.

Methods We evaluated 2048 consecutive patients who underwent successful DES implantation. The primary outcomes were stent thrombosis, death, myocardial infarction (MI), and target vessel revascularisation.

Results After 2 years of follow-up, there were 11 stent thrombosis, 72 deaths, 113 MI, 133TVR. In multivariable Cox proportionalhazards models, the high CRP remained predictive of adverse cardiac events, elevated levels of CRP were significantly associated with increased risks of stent thrombosis (HR 4.08; 95\% CI 1.91 to 11.44; $\mathrm{p}<0.001$ ), death (HR 2.01; 95\% CI 1.18 to 4.688; $\mathrm{p}=0.004$ ), MI (HR 1.78 ; $95 \%$ CI 1.25 to $9.12 ; \mathrm{p}=0.001)$, but not target vessel revascularisation (HR 1.20; 95\% CI 0.59 to 1.16; $\mathrm{p}=0.62$ ).

Conclusions Elevated CRP levels were significantly associated with major coronary events after DES implantation, such as stent thrombosis, death, and MI, demonstrating the additive impacts of active inflammation and myocardial injury on prognosis after DES implantation.

\section{e0421 DIAGNOSTIC VALUE OF PLASMA SRAGE IN CORONARY ARTERY DISEASE AND CORONARY ARTERY DISEASE WITH ACUTE MYOCARDIAL INFARCTION (AMI)}

doi:10.1136/hrt.2010.208967.421

${ }^{1}$ Guo Caixia, 'Zhu Xinyuan, ${ }^{2}$ Zhang Like, ${ }^{1}$ Song Ke, ${ }^{1}$ Xu Xiaowei, ${ }^{1} \mathrm{Du}$ Fenghe. ${ }^{1}$ Department of Cardiology Medicine, Beijing Tiantan Hospital Affiliate of Capital University of Medical Sciences, Beijing, China; ${ }^{2}$ Department of Pathophysiology, Capital University of Medical Sciences, Beijing, China

Objective To elucidate whether plasma soluble receptor for advanced glycation end products (sRAGE) is the biochemical markers indicating coronary artery disease and coronary artery disease with acute myocardial infarction (AMI).

Method Plasma levels of sRAGE was determined by enzyme linked immunoabsorbent assay in patients who came from Cardiology Department of Tiantan Hospital form March to May 2009 categorised as group I (non-CAD subjects), group II (CAD without myocardial infarction subjects), and group III (CAD with AMI subjects).

Results Plasma levels of sRAGE was higher in group III than in group II $(p<0.01)$ and in group I $(p<0.01)$. The levels of sRAGE between group II and group I were of no statistical difference $(p>0.05)$. Further more, to evaluate whether sRAGE is the biochemical markers indicating the AMI in CAD patients, ROC curve was used, and area under the curve was $0.855(\mathrm{p}=0.000)$.

Conclusion sRAGE may be new biochemical markers indicating AMI in the patients with coronary artery disease. The diagnostic sensitivity of sRAGE is $75 \%$ and specificity is $86.4 \%$.

\section{e0422 DIAGNOSTIC VALUE OF ADENOSINE STRESS ${ }^{\text {99M}}$ TC-MIBI GATED MYOCARDIAL PERFUSION IMAGING FOR CORONARY ARTERY DISEASE}

doi:10.1136/hrt.2010.208967.422

${ }^{1}$ Wang Lijuan, ${ }^{1} \mathrm{Li}$ Xiaojuan, ${ }^{1}$ Sun Yingxian, ${ }^{2} \mathrm{Li} \mathrm{Na},{ }^{2} \mathrm{Li}$ Yaming. ${ }^{1}$ Department of Cardiology The First Affiliated Hospital of China Medical University Shenyang, China; ${ }^{2}$ Department of Nuclear Medicine The First Affiliated Hospital of China Medical University Shenyang, China

Objective Stress ${ }^{99 \mathrm{~m}}$ Tc-MIBI myocardial perfusion imaging (MPI)) be useful in evaluating myocardial ischaemia and judging coronary artery stenosis. The purpose of this study was to evaluate the sensitivity, specificity and accuracy of adenosine stress ${ }^{99 \mathrm{~m}} \mathrm{Tc}-\mathrm{MIBI}$ gated myocardial perfusion SPECT (G-MPI) for the diagnosis of coronary artery disease (CAD).

Materials and methods The subjects were forty-six patients diagnosed or suspected $\mathrm{CAD}$, including forty with angina, six with old myocardial infarction. Adenosine stress G-MPI were performed 90 min after injection of adenosine, and resting G-MPI performed in same day. All patients underwent coronary artery angiography within two weeks. Significant stenosis was defined when the coronary artery intraluminal stenosis $\geq 50 \%$. Analyse the imaging and calculate the sensitivity, specificity and accuracy of adenosine stress G-MPI in diagnosing CAD and judging coronary artery stenosis. Do correlation analysis of left ventricular systolic function (left ventricular ejection fraction) between adenosine stress ${ }^{99 m}$ Tc-MIBI G-MPI and echocardiography.

Results The sensitivity, specificity, accuracy, positive predictive value and negative predictive value of adenosine stress ${ }^{99 \mathrm{~m}} \mathrm{Tc}-\mathrm{MIBI}$ G-MPI for the diagnosis of CAD were $87.0 \%, 85.7 \%, 86.6 \%, 93.8 \%$, and $72.7 \%$ respectively. The sensitivity, specificity and accuracy for the diagnosis of LAD, LCX and RCA stenosis were $88.2 \%, 90.9 \%$, $88.9 \% ; 78.6 \%, 83.3 \%, 80.0 \% ; 90.5 \%, 81.8 \%, 87.5 \%$ respectively. LVEF-G-MPI correlated with LVEF-UCG significantly, with a correlation coefficient of $0.885(\mathrm{R}=0.0001)$.

Conclusions In Conclusion, stress adenosine ${ }^{99 \mathrm{~m}} \mathrm{Tc}-\mathrm{MIBI}$ G-MPI have provided better sensitivity, specificity and accuracy in the diagnosis of $\mathrm{CAD}$, and is probably an accurate method for detecting coronary artery branch stenosis. It can be used in evaluating left ventricular function, especially for patients unsuited in the exercise MPI.

\section{e0423 THE RESEARCH ABOUT THE SHORT-TIME EFFECTS OF PREINFRACTION ANGINA IN PATIENTS COMPLICATED WITH ACUTE MYOCARDIAL INFRACTION AND TREATED BY PRIMARY PERCUTANEOUS CORONARY INTERVENTION}

doi:10.1136/hrt.2010.208967.423

Cao Rui Caoxuebin. 252 Hospital of Baoding, Hebei, China

Objective To evaluate the short-time effects, angiographic characteristics, and the clinical outcome of preinfarction angina in patients with first acute myocardial infarction (AMI) after emergency primary percutaneous coronary intervention (PCI). And evaluate the influence of diabetes mellitus to preinfarction angina.

Methods The clinical data of 130 patients with AMI hospitalised from January 2006 to December 2008, who underwent emergency 